\title{
Efficacy of Montelukast and Levocetirizine as Treatment for Allergic Rhinitis
}

\author{
Vipan Gupta ${ }^{1}$ and Prithpal Singh Matreja ${ }^{2 *}$ \\ ${ }^{1}$ Department of Otorhinolaryngology, Gian Sagar Medical College, Village Ram Nagar, Tehsil Rajpura, District Patiala, Punjab, India -140601 \\ ${ }^{2}$ Department of Pharmacology, Gian Sagar Medical College, Village Ram Nagar, Tehsil Rajpura, District Patiala, Punjab, India -140601
}

\section{Abstract}

Antihistamines are effective in reducing majority of symptoms of allergic rhinitis, but are ineffective for nasal congestion and nighttime symptoms. Montelukast have been found to provide quick relief. Comparison of Montelukast has been done with Antihistamines but data is limited. Hence, this study was done to compare the effectiveness of montelukast combined with levocetirizine once daily to levocetirizine alone for a 6-week treatment course of allergic rhinitis. In this randomized, open, parallel study, out of 102 patients were randomly assigned to receive montelukast and levocetirizine (treatment group) or levocetirizine alone (control group), 95 patients completed the entire 6 weeks of study. The primary outcome measure was the mean change of the total daytime nasal symptom score (PDTS) and secondary outcome measures were mean change of night time nasal, daytime eye and composite symptom (PNTS, PES, PCS). The change in total daytime nasal symptom, composite symptoms and nighttime nasal symptom scores was significantly $(p<0.05)$ greater in montelukast and levocetirizine group than in levocetirizine alone group. The change in daytime eye symptom scores was comparable in both the groups but not statistically significant $(p=0.94)$. Montelukast combined with levocetirizine was effective in reducing daytime, nighttime, composite and daytime eye symptom score as compared to levocetrizine alone.
\end{abstract}

Keywords: Allergic rhinitis; Antihistamines; Montelukast; Levocetirizine

Abbreviations: PDTS: Daytime Nasal Symptom Score; PNTS: Nighttime Nasal Symptom Score; PES: Daytime Eye Symptom Score; PCS: Composite Symptom Score

\section{Introduction}

Allergic rhinitis is the most common atopic disorder affecting $18 \%$ to $40 \%$ of adults worldwide, diagnosed by history, physical exam and objective testing [1]. According to the Allergic Rhinitis and its Impact on Asthma (ARIA) document it is classified by chronicity (intermittent or persistent), and severity which is based on symptoms and quality of life (mild, or moderate/ severe). The terms "seasonal" and "perennial" allergic rhinitis were previously categorized as allergic rhinitis by the clinically significant aeroallergen. Perennial allergic rhinitis is associated with year round and indoor allergens including mold spores, cockroaches, dust mite fecal particles, animal dander, and occupational exposure. Seasonal allergic rhinitis is commonly referred to as "hay fever", developing during a defined pollen season, and is usually intermittent, as a result of allergic reactions to outdoor aeroallergens including mold spores, and pollens of trees, grasses, and weeds that depend on wind for cross-pollination. Commonly there is an overlap of "perennial" and "seasonal" symptoms in some geographic regions which has resulted in decreased use and confusion regarding these terms $[2,3]$.

On physical examination, patients classically can have pale nasal mucosa with swollen, oedematous turbinate's and clear nasal secretions (rhinorrhea). A thorough history and physical are sufficient to make a preliminary diagnosis and initiate treatment. Role of diagnostic nasal endoscopy and radiological investigation is important for assessing ventilation of sinuses, any polyp formation obstructing the osteomeatal complex and thus helping in making a decision for appropriate management of the case. Specific immunoglobulin $E$ (IgE) antibodies can be demonstrated by either skin testing or in vitro radioallergosorbent (RAST) testing to which a person has become sensitized may be necessary in patients with difficult to treat allergic rhinitis [2-4].
Usually patients are made aware of the fact that allergic symptoms can be controlled and cure is only limited with fair chance of recurrence. Treatment of allergic rhinitis depends upon several factors. The first involves avoidance of implicated allergens. Unfortunately, the effort to appropriately reduce levels of indoor allergens is often too difficult for patients to accomplish and even more difficult is the prevention of exposure to outdoor allergens $[3,4]$.

Drug therapy for allergic rhinitis should be guided by the type and severity of individual patient's symptoms and should reduce nasal congestion, sneezing, and rhinorrhea over the course of the entire day and night and physician preferences [3,5]. Pharmacotherapy includes oral and intranasal $\mathrm{H}_{1}$ antihistamines, intranasal corticosteroids, oral and intranasal decongestants, intranasal anticholinergics, intranasal cromolyn and leukotriene receptor antagonists [2-4].

Antihistamines are effective in reducing pruritis, sneezing and watery rhinorrhea, and are a mainstay therapy for allergic rhinitis [6]. Although first generation antihistamines are generally more effective in controlling rhinorrhea compared with second generation antihistamines, their use is markedly limited due to greater anticholinergic effects $[7,8]$. Second generation antihistamines have shown favourable effect on sleep in patients with allergic rhinitis $[5,9,10]$ and are in general recommended for mild to moderate disease as first-line therapy, but not effective in nasal congestion [3].

*Corresponding author: Prithpal Singh Matreja, Assistant Professor, Department of Pharmacology, Gian Sagar Medical College, Village Ram Nagar, Tehsil Rajpura District Patiala, Punjab, India -140601, Tel: +91-9855001847; Fax: +91-1762520024; E-mail: drpsmatreja@yahoo.co.in

Received October 03, 2010; Accepted October 19, 2010; Published October 20 2010

Citation: Gupta V, Matreja PS (2010) Efficacy of Montelukast and Levocetirizine as Treatment for Allergic Rhinitis. J Aller Ther 1:103. doi:10.4172/2155-6121.1000103

Copyright: ( 2010 Gupta V, et al. This is an open-access article distributed under the terms of the Creative Commons Attribution License, which permits unrestricted use, distribution, and reproduction in any medium, provided the original author and source are credited. 
Montelukast serves a role in helping reduce symptoms of allergic rhinitis that are not controlled with antihistamines alone by competitively and reversibly inhibits cysteinyl leukotrienes (CysLTs), specifically leukotrienes D4 (LTD4), theoretically decreasing congestion and stuffiness associated with allergic rhinitis [4]. Montelukast, as monotherapy has been effective in improving daytime and nighttime symptoms in patients with allergic rhinitis $[3,11]$ and in comparison to antihistamines appear to have significantly better improvement in night time symptoms [12-14].

Hence, a combination therapy of montelukast with antihistamines could provide enhancing and complementary effects, thereby reducing both the daytime and night time symptoms effectively. Combination of levocetirizine with montelukast has shown a significant improvement in patients with allergic rhinitis. There was a significant improvement in both daytime and nighttime symptoms in patients on combination therapy as compared to placebo and giving both the drugs as monotherapy [15-17]. There are only limited studies available for the effect of combination therapy of montelukast and levocetirizine on the Indian population. Hence, this study was designed to assess the efficacy of montelukast with levocetirizine as treatment for allergic rhinitis in Indian population.

\section{Materials and Methods}

\section{Study design}

This prospective, randomized, open, parallel group study (with a 2 week run-in period and a 6 week treatment period) was conducted between July 2008 to April 2010 at the outpatient department of Gian Sagar Medical College and Hospital, District Patiala. Clinic visits were scheduled at screening (visit 1), after a 14 day run-in period (visit 2), after every 2 weeks of treatment according to randomization for 6 weeks (visit 3,4,5). During the run-in period patients received only Tripolidine $\mathrm{HCl}$ and Pseudoephedrine $\mathrm{HCl}$ as needed to relieve symptoms. During the randomization period patients were randomly allocated using random number table to receive Tab Levocetirizine $5 \mathrm{mg}$ and Montelukast $10 \mathrm{mg}$ in the treatment group or levocetirizine $5 \mathrm{mg}$ in control group once daily at bed time for 6 weeks. The medical compliance was determined from the returned tablet count. A physical examination for nasal secretion and turbinate swelling was also done at each visit.

The study protocol and informed consent were reviewed and approved by Institutional Ethics Committee of Gian Sagar Medical College and Hospital before the study initiation and written informed consent was obtained from each patient prior to enrolment in the study. The study was conducted in accordance with ICH-GCP guidelines.

\section{Patients}

Adults 18-60 years old of both sexes with a clinical history of perennial allergic rhinitis for at least 1 year, non-smokers, who could read and understand the protocol (middle level education) and were willing to give written consent, were eligible for study. Study exclusion included pregnancy or lactation, any polyposis or infectious pathology during physical examination, alcohol or illicit drug, asthma, major surgery within 4 weeks, upper respiratory tract infection within 3 weeks prior to study.

Medications that were prohibited prior to the study included: nasal or inhaled corticosteroids within 2 weeks, oral corticosteroids within 1 month, cetrizine, ketotifen, loratidine, oral or inhaled long acting beta agonist or inhaled anticholinergics within 1 week and levocetirizine or fexofenatidine within 72 hours.

\section{Daily rhinitis diary card}

Recorded on the daily diary card, the allergic rhinitis and conjunctivitis symptoms were assessed on a 4-point scale (0 to 3 ) for both daytime (diary card completed in the evening) and nighttime (diary card completed on awakening). The daytime nasal (rhinorrhea, sneezing, itching, and congestion), night time nasal (nasal congestion upon awakening, difficulty going to sleep, and night time awakening) and eye (tearing, itching, redness and puffing) symptoms and their rating were described to every patient by the same technician. The ratings of the symptom were: $0=$ not noticeable, $1=$ mild symptoms, $2=$ moderate symptoms, $3=$ severe symptoms. The rating had to be performed by the patients themselves to increase the creditability of the subjective scale. Each dose of Tripolidine $\mathrm{HCl}$ and Pseudoephedrine $\mathrm{HCl}$ was scored as scale 2 and was included in the total daytime nasal symptom scores of the run-in and randomization periods.

Safety evaluation included spontaneously reported adverse events throughout the study.

\section{Outcome measurements}

The primary outcome measure was the mean change of the total daytime nasal symptom scores (PDTS), defined as the average score of four daytime nasal symptoms.

The secondary outcomes were the mean changes of the nighttime nasal symptom scores (PNTS), daytime eye symptom scores (PES), composite symptom scores (PCS) (average score of day and nighttime nasal symptom score). The credibility of the nasal examinations of the subjects was markedly enhanced by the single-observer design of this trial for every patient, which eliminated the inter-observer reliability issue.

\section{Statistical analysis}

The data was tabulated as mean \pm standard deviation (SD). Results were analyzed using non parametric tests (Chi-Square Test, Wilcoxon Sign Ranked Test and Mann Whitney U Test) and parametric tests (two tailed student t-test). A $\mathrm{p}<0.05$ was considered statistically significant.

Nominal variables were compared with Chi-square analysis. The Student-t test was used for comparison of group means for normally distributed data and the Mann-Whitney U test/ Wilcoxon Sign Rank Test was used for non-normally distributed data.

\section{Results}

\section{Patients}

Of 250 screened patients, 122 patients were enrolled in the runin period. Absence of reactivity to 1 or more allergens and infections were the most common reasons for excluding patients from the run-in period. 10 patients from each group were withdrawn from the study due to protocol deviation, or infection. 7 patients out of 102 enrolled did not complete the entire follow up. 2 patients (1 from each group) were lost to follow-up after 2 weeks of therapy and 5 patients ( 3 patients in treatment group and 2 patients in control group) were lost to follow up after 4 weeks of therapy. 95 patients completed the entire 6 weeks follow-up of the study. 


\section{Efficacy}

The patients in both groups had comparable demographic and clinical profile as shown in (Table 1).

The PDTS, PNTS, PCS and PES scores reduced significantly as compared to baseline in both groups. PDTS score (Mean \pm SD) at baseline was $2.055 \pm 0.329$ which reduced significantly to $1.18 \pm 0.437$ at the end of 6 weeks in treatment group. Similarly, PDTS score reduced significantly from $2.045 \pm 0.318$ to $1.57 \pm 0.471$ at the end of 6 weeks in control group (Figure 1). The PNTS score (Mean \pm SD) decreased significantly from $1.93 \pm 0.369$ to $1.14 \pm 0.448$ in treatment group and from $1.93 \pm 0.487$ to $1.34 \pm 0.489$ in control group at the end of 6 weeks (Figure 2). The PCS score (Mean \pm SD) decreased significantly from $1.99 \pm 0.246$ to $1.17 \pm 0.402$ in treatment group and from $1.985 \pm 0.279$ to $1.46 \pm 0.41$ in control group at the end of 6 weeks (Figure 3). The PES score (Mean \pm SD) decreased significantly from $2.01 \pm 0.262$ to $1.37 \pm 0.48$ in treatment group and from $2.02 \pm 0.404$ to $1.40 \pm 0.455$ in control group at the end of 6 weeks.

The improvement in treatment group was significantly $(\mathrm{p}<0.05)$ more as compared to control group from $4^{\text {th }}$ week onwards in PDTS score $(1.63 \pm 0.395$ Vs $1.81 \pm 0.383)$, PCS scores $(1.57 \pm 0.328$ Vs $1.73 \pm 0.337)$ and at $6^{\text {th }}$ weeks in PNTS score $(1.14 \pm 0.448$ Vs $1.34 \pm 0.489$ ). There was no significant difference in the PES score in both the groups during the entire duration of treatment.

\section{Safety}

There was no serious adverse event reported in both groups. The incidence of adverse event reported in treatment group was more as compared to control group but none of the adverse event

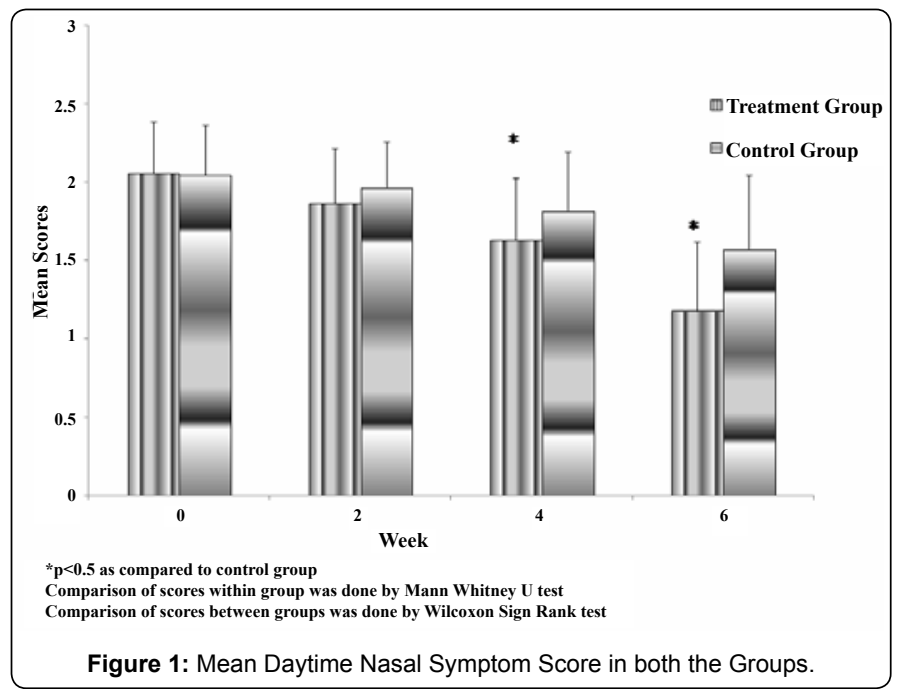

reported was severe that required termination of treatment. The adverse event reported in both groups did not require reduction in dose or any therapy for treatment of adverse events. Patients in both groups reported with nausea, dizziness, fatigue, headache, somnolence, restlessness, dry mouth, fever and weakness. There was no prolongation of hospitalization in any patient.

\section{Discussion}

Allergic rhinitis is the most common allergic disease affecting the general population worldwide [1], leading to inflammation of the upper airway mucous membranes due to binding of antigens to specific IgE. According to a recent survey of 2,500 adults diagnosed with allergic rhinitis the most bothersome symptoms for patients are nasal congestion, runny nose, postnasal drip, red itchy eyes, and headaches [18]. A vicious cycle begins with nasal congestion, which elicits breathing through the mouth, difficulties in falling asleep, nighttime awakening, and snoring, nasal congestion on awakening with consequent daytime somnolence, impaired mood, poor memory and decreased productivity at school and work. In due course of time, if therapy is not given a chronic state of nasal inflammation accompanied by nasal obstruction can develop and lead to sinusitis, otitis media with effusion, nasal polyps, and asthma [1].

Combination of montelukast with antihistamines is well tolerated and has shown equivocal results [3]. In the present study, Montelukast with levocetirizine was very effective in improving PDTS scores in patients of allergic rhinitis. Therapy with Montelukast with levocetirizine also significantly improved PNTS and PCS scores as compared to levocetirizine alone.

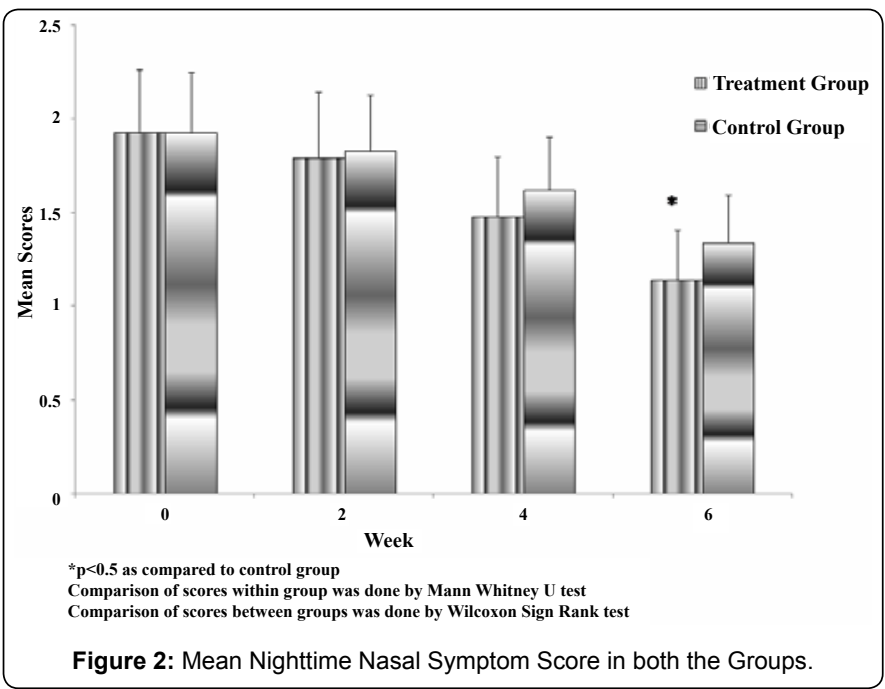

\begin{tabular}{|l|l|l|}
\hline Characteristics & Control group & Treatment group \\
\hline $\begin{array}{l}\text { No. patients } \\
\text { Fale }\end{array}$ & $\begin{array}{l}\mathrm{n}=47 \\
25\end{array}$ & $\mathrm{n}=48$ \\
27 & 22 & 21 \\
\hline Age, years (mean \pm SD) & $35.3 \pm 12.62$ & $35.47 \pm 11.93$ \\
\hline Mean Daytime nasal symptom score (mean \pm SD) & $2.055 \pm 0.33$ & $2.045 \pm 0.32$ \\
\hline Mean Nightime nasal symptom score (mean \pm SD) & $1.93 \pm 0.37$ & $1.927 \pm 0.49$ \\
\hline Mean Composite symptom score (mean \pm SD) & $1.99 \pm 0.25$ & $1.985 \pm 0.30$ \\
\hline Mean Daytime eye symptom score (mean \pm SD) & $2.01 \pm 0.26$ & $0.90^{*}$ \\
\hline
\end{tabular}

\# Chi-Square Test

* Unpaired t-test

† Mann Whitney U Test

Table 1: Baseline characteristics of the study group. 


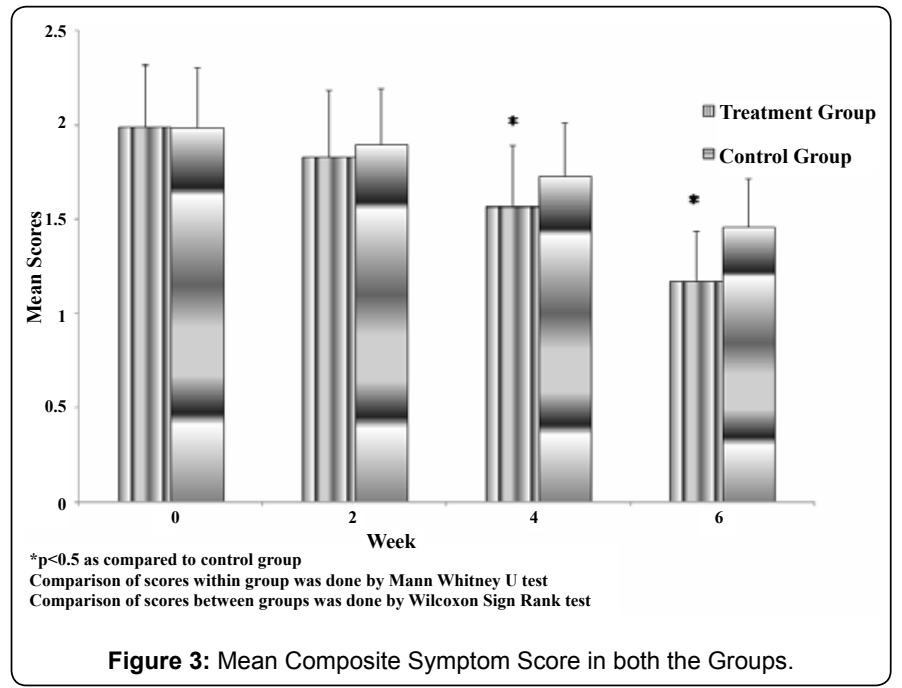

The results are in agreement with earlier studies that have demonstrated a significant improvement in the nasal symptoms of patients suffering from allergic rhinitis in patients receiving montelukast and levocetirizine $[15,16]$. While the results of our study are different for other study which showed that combination treatment significantly reduced the eye symptoms as compared to monotherapy [17].

Montelukast, a leukotriene receptor antagonist has been studied quite extensively in the treatment of allergic rhinitis over the past few years as monotherapy, combined with a second generation antihistamine, and with or without intranasal corticosteroids. Some studies have found a significant improvement in the daytime nasal symptoms scores, daytime eye symptom scores, nighttime symptom scores and composite symptom scores when combined with an antihistaminic drug. There has been a statistically significant reduction in the nighttime symptoms (difficulty falling asleep, nighttime awakenings and congestion upon awaking) appeared with montelukast. Studies have even shown a significant improvement in nasal congestion during both allergen challenge and the recovery phase $[3,4,19]$.

The most common adverse effect reported in our study with montelukast and levocetirizine was nausea, dizziness, fatigue, headache, somnolence, restlessness, dry mouth, fever and weakness, whereas in the control group patients reported of fatigue, somnolence, restlessness, dry mouth, fever and weakness. The adverse effects reported are similar to those reported in earlier studies [15]. None of the adverse event reported was severe that required termination of treatment or reduction in dose or any therapy for treatment of adverse events.

These results confirm and extend earlier finding, that montelukast, a leukotriene receptor antagonist with levocetirizine is effective and safe in Indian patients of allergic rhinitis and may be of more clinical utility in alleviation of residual symptoms and improvement of quality of life associated with allergic rhinitis $[3,4]$.

Limitation of our study are, firstly the sample size is small so the number of adverse event reported are not significantly more as compared to levocetirizine alone, may be a larger sample size could show significant difference. Secondly, this is an open-label study due to non availability of funds, a double-blind study would have been ideal.

To conclude, patients in both groups tolerated the treatment. There was significant decrease in PDTS, PNTS, PCS and PES score in both groups as compared to baseline. However, Patients treated with montelukast and levocetirizine had earlier response and the relief of residual symptoms was more as compared to conventional treatment.

\section{Competing Interest}

Neither the author nor the co-author has received any financial or non-financial help from any other source.

\section{References}

1. Woods L, Craig TJ (2006) The importance of rhinitis on sleep, daytime somnolence, productivity and fatigue. Curr Opin Pulm Med 12: 390-396.

2. Bousquet J, Lund VJ, van Cauwenberge P, Bremard-Oury C, Mounedji N, et al. (2003) Implementation of guidelines for seasonal allergic rhinitis: a randomized controlled trial. Allergy 58: 733-741.

3. Lagos JA, Marshall GD (2007) Montelukast in the management of allergic rhinitis. Ther Clin Risk Manag 3: 327-332.

4. Gonyeau MJ, Partisano AM (2003) A clinical review of montelukast in the treatment of seasonal allergic rhinitis. Formulary 38: 368-378.

5. Storms W (2008) Allergic rhinitis-induced nasal congestion: its impact on sleep quality. Prim Care Resp J 17: 7-18.

6. BousquetJ, van Cauwenberge P, Khaltaev N (2001) Allergic rhinitis and its impact on Asthma. J Allergy Clin Immunol 108: 147-336.

7. Camelo-Nunes IC (2006) New antihistamines: a critical review. J Pediatr (Rio J) 82: S173-S180

8. Brunton LL, Lazo JS, Parker KL (2006) Histamine, Bradykinin and Thei antagonist: Goodman and Gilman's The pharmacological Basis of Therapeutics. (11th edn) New York: McGraw-Hill.

9. Meltzer EO, Prenner BM, Nayak A, Desloratadine Study Group (2001) Efficacy and tolerability of once-daily $5 \mathrm{mg}$ desloratadine, an $\mathrm{H}_{1}$ receptor antagonist, in patients with seasonal allergic rhinitis. Clin Drug Invest 21: 25-32.

10. De Blic J, Wahn U, Billard E, Alt R, Pujazon MC (2005) Levocetirizine in children evidenced efficacy and safety in a 6-week randomized seasonal allergic rhinitis trial. Pediatr Allergy and Immunol 16: 267-275.

11. Patel P, Philip G, Yang W, Call R, Horak F, et al. (2005) Randomized, doubleblind, placebo-controlled study of montelukast for treating perennial allergic rhinitis. Ann Allergy Asthma Immunol 95: 551-557.

12. Philip G, Malmstrom K, Hampel FC, Weinstein SF, LaForce CF, et al. (2002) Montelukast for treating seasonal allergic rhinitis: a randomized, double blind, placebo-controlled trial performed in the spring. Clin Exp Allergy 32: 1020-1028.

13. Nayak AS, Philip G, Lu S, Malice MP, Reiss TF, et al. (2002) Efficacy and tolerability of montelukast alone or in combination with loratadine in seasonal allergic rhinitis: a multicenter, randomized, double blind, placebo controlled trial performed in the fall. Ann Allergy Asthma Immunol 88: 592-600.

14. van Adelsberg J, Philip G, LaForce CF, Weinstein SF, Menten J, et al. (2003) Randomized controlled trial evaluating clinical benefit of montelukast for treating spring seasonal allergic rhinitis. Ann Allergy Asthma Immunol 90: 214222

15. Ciebiada M, Ciebiada MG, Kmieck T, Dumuske LM, Gorski P (2008) Quality of life in patients with persistent allergic rhinitis treated with montelukast alone or in combination with levocetirizine or desloratidine. J Invest Allergol Clin Immunol 18: 343-349.

16. Ciebiada M, Ciebiada MG, Dubuske LM, Gorski P (2006) Montelukast with desloratidine or levocetirizine for the treatment of persistent allergic rhinitis. Ann Allergy Asthma Immunol 5: 664-671.

17. Kurowski M, Kuna P, Gorski P (2004) Montelukast plus cetirizine in prophylactic treatment of seasonal allergic rhinitis: influence on clinical symptoms and nasa allergic inflammation. Allergy 59: 280-288.

18. Allergies in America: A landmark survey of nasal allergy sufferers: Executive Summary.

19. Watanasomsiri A, Paochanukoon O, Vichyanond P (2008) Efficacy of Montelukast and loratidine as treatment for allergic rhinitis in children. Asian Pac J Allergy and Immunol 26: 89-95. 